QUARTERLY OF APPLIED MATHEMATICS

VOLUME LXIV, NUMBER 1

MARCH 2006, PAGES 153-165

$\mathrm{S}$ 0033-569X(06)00992-1

Article electronically published on January 24, 2006

\title{
SH-WAVES IN LAMINATED PLATES
}

\author{
BY \\ SERGEY V. KUZNETSOV \\ Institute for Problems in Mechanics, Prosp. Vernadskogo, 101, Moscow, 119526 Russia
}

\begin{abstract}
A mathematical model for analyzing horizontally polarized shear surface (SH) waves propagating in laminated plates is presented. All the layers are assumed to have monoclinic symmetry. Three types of boundary conditions imposed on the outer surfaces of a plate are considered. A variant of the modified transfer matrix (MTM) method is developed. Closed form dispersion relations are obtained for plates consisting of one or two orthotropic layers. Asymptotic solutions for orthotropic two- and threelayered plates are derived.
\end{abstract}

1. Introduction. In our previous paper [1 the Modified Transfer Matrix (MTM) method was worked out for analysis of Love waves propagating in a stratified heterogeneous medium consisting of monoclinic layers lying on a monoclinic half-space (substrate). As is natural for analyses of all kinds of surface waves propagating in semibounded media, the following attenuation condition for a surface wave in a half-space was imposed:

$$
\mathbf{u}(\mathbf{x}, t)=O\left(\left|x^{\prime}\right|^{-1}\right), \quad\left|x^{\prime}\right| \rightarrow \infty,
$$

where $\mathbf{u}$ is the displacement field in the substrate, $x^{\prime} \equiv \nu \cdot \mathbf{x}$ is the coordinate along depth of the substrate, and $\nu$ is the unit normal to the plane boundary of the substrate. Condition (1) resembles Sommerfeld's emission condition, but is applied only to a specific direction determined by vector $\nu$. This condition eliminates the so-called leakage partial waves, leading to the unbounded at $\left|x^{\prime}\right| \rightarrow \infty$ displacement and stress fields.

Herein, the MTM method is applied to analysis of horizontally polarized shear surface waves propagating in laminated plates consisting of anisotropic layers, all of which have the same plane of elastic symmetry. The outer surfaces of the plates are assumed to be either clamped, traction-free, or having mixed boundary conditions (one outer surface is clamped, and another is traction-free). Thus, the regarded waves differ from Love waves [2] in absence of the contacting substrate and, hence, lack of necessity in satisfying condition (1). From a terminological point of view, these waves can be referred to as

Received June 7, 2005.

2000 Mathematics Subject Classification. Primary 74J15.

Key words and phrases. SH-wave, shear wave, surface wave, love wave, anisotropy, laminated plate.

E-mail address: svkuznec@ipmnet.ru

(C)2006 Brown University 
the SH or shear Lamb waves, while the genuine Lamb waves [3] generally have elliptic polarization and propagate in a plate with the traction-free outer surfaces. See also [4, where Lamb waves in a homogeneous anisotropic plate with arbitrary elastic anisotropy and different boundary conditions are analyzed by applying the Stroh six-dimensional formalism. The MTM method appears to be rather fast, as is typical for all realizations of the Transfer Matrix (TM) methods (see [1] for discussions), and at the same time it possesses good numerical stability due to (i) choosing floating origins for the coordinate systems in layers, and (ii) using a modified representation for the surface waves in layers based on use of hyperbolic functions.

As will be demonstrated, the MTM method can be applied to obtaining analytical solutions for plates containing two or three layers. It will also be shown that under certain conditions depending on the phase speed and material properties of a necessary anisotropic layer, the traditional representation for the considered surface waves becomes invalid, and the correct representation will be constructed.

2. Basic notations. In the subsequent analysis all layers of the plate are assumed to be homogeneous and linearly hyperelastic. Equations of motion for homogeneous anisotropic elastic medium can be written in the form

$$
\mathbf{A}\left(\partial_{x}, \partial_{t}\right) \mathbf{u} \equiv \operatorname{div}_{x} \mathbf{C} \cdots \nabla_{x} \mathbf{u}-\rho \ddot{\mathbf{u}}=0,
$$

where the elasticity tensor $\mathbf{C}$ is assumed to be positive definite:

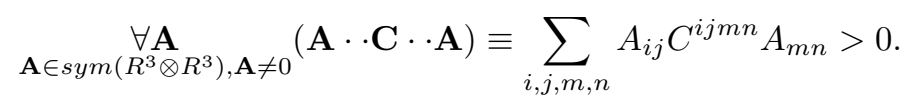

REMARK 2.1. a) The other assumption concerns symmetry of the elasticity tensor. It will be assumed that all the regarded materials possess planes of elastic symmetry coinciding with the sagittal plane $\mathbf{m} \cdot \mathbf{x}=0$, where vector $\mathbf{m}$ is the polarization vector. This is achieved by the elasticity tensor belonging to the monoclinic system; the latter is equivalent to vanishing all of the decomposable components of the tensor $\mathbf{C}$ having an odd number of entries of the vector $\mathbf{m}$ in the orthogonal basis in $R^{3}$ generated by the vector $\mathbf{m}$ and any two orthogonal vectors belonging to the sagittal plane.

b) It will be shown later that assuming monoclinic symmetry provides a sufficient condition for the surface tractions acting on any plane $\nu \cdot \mathbf{x}=$ const to be collinear with vector $\mathbf{m}$.

Following [1], we will seek a horizontally polarized shear wave in a layer in the form

$$
\mathbf{u}(\mathbf{x})=\mathbf{m} f\left(i r x^{\prime}\right) e^{i r(\mathbf{n} \cdot \mathbf{x}-c t)},
$$

where the coordinate $x^{\prime}=\nu \cdot \mathbf{x}$ is as defined in (1); $f$ is the unknown scalar function; the exponential multiplier in (4) corresponds to propagation of the plane wave front along direction $\mathbf{n}$ with the phase speed $c$; and $r$ is the wave number. Substituting representation (4) into Eq. (2) and taking into account Remark 2.1a yields the following differential equation:

$$
\left.\begin{array}{r}
(\mathbf{m} \otimes \nu \cdots \mathbf{C} \cdot \nu \otimes \mathbf{m}) f^{\prime}+(\mathbf{m} \cdot \operatorname{sym}(\nu \cdot \mathbf{C} \cdot \mathbf{n}) \cdot \mathbf{m}) f^{\prime} \\
+\left(\mathbf{m} \otimes \mathbf{n} \cdot \mathbf{C} \cdot \mathbf{n} \otimes \mathbf{m}-\rho c^{2}\right) f
\end{array}\right)=0 .
$$


A characteristic equation for the differential equation (5), known also as the Christoffel equation, has the form

$$
\left.\begin{array}{r}
(\mathbf{m} \otimes \nu \cdots \mathbf{C} \cdot \nu \otimes \mathbf{m}) \gamma^{2}+(\mathbf{m} \cdot \operatorname{sym}(\nu \cdot \mathbf{C} \cdot \mathbf{n}) \cdot \mathbf{m}) \gamma \\
+\left(\mathbf{m} \otimes \mathbf{n} \cdot \mathbf{C} \cdot \mathbf{n} \otimes \mathbf{m}-\rho c^{2}\right)
\end{array}\right)=0 .
$$

The left-hand side of Eq. (6) represents a polynomial of degree 2 with respect to the Christoffel parameter $\gamma$. Thus, for the regarded elastic symmetry only two partial waves form the regarded $\mathrm{SH}$-wave in a layer.

3. Displacements and surface tractions in layers. In this section the lower index $k(1 \leq k \leq n)$ is referred to as the corresponding layer in the $n$-layered plate. It flows out from analysis of Eq. (5) that the structure of the solution depends upon multiplicity of the roots of Eq. (6). The following two cases are possible.

3.1. Aliquant roots. For aliquant roots of the Cristoffel equation, the solution for the displacement field can be represented in the form

$$
\mathbf{u}_{k}(\mathbf{x})=\mathbf{m}\left(C_{2 k-1} \sinh \left(i r \alpha_{k} x^{\prime}\right)+C_{2 k} \cosh \left(i r \alpha_{k} x^{\prime}\right)\right) e^{i r\left(\beta_{k} x^{\prime}+\mathbf{n} \cdot \mathbf{x}-c t\right)},
$$

where for convenience of the subsequent analysis the corresponding root $\gamma_{k}$ is represented in the form $\gamma_{k}=\alpha_{k}+\beta_{k}$; herein $\alpha_{k}$ is the discriminant of Eq. (6), and

$$
\begin{aligned}
& \alpha_{k}= \pm \sqrt{\left(\frac{\mathbf{m} \cdot \operatorname{sym}\left(\mathbf{n} \cdot \mathbf{C}_{k} \cdot \nu\right) \cdot \mathbf{m}}{\mathbf{m} \otimes \nu \cdot \cdot \mathbf{C}_{k} \cdot \nu \otimes \mathbf{m}}\right)^{2}-\frac{\mathbf{m} \otimes \mathbf{n} \cdot \mathbf{C}_{k} \cdot \cdot \mathbf{n} \otimes \mathbf{m}-\rho_{k} c^{2}}{\mathbf{m} \otimes \nu \cdot \cdot \mathbf{C}_{k} \cdot \nu \otimes \mathbf{m}}}, \\
& \beta_{k}=-\frac{\mathbf{m} \cdot \operatorname{sym}\left(\mathbf{n} \cdot \mathbf{C}_{k} \cdot \nu\right) \cdot \mathbf{m}}{\mathbf{m} \otimes \nu \cdot \mathbf{C}_{k} \cdot \cdot \nu \otimes \mathbf{m}}
\end{aligned}
$$

where $\operatorname{sym}(\mathbf{A}) \equiv \frac{1}{2}\left(\mathbf{A}+\mathbf{A}^{t}\right)$ for any second-order tensor $\mathbf{A}$. Thus, the parameter $\alpha_{k}$ is either real or imaginary depending on the value of the phase speed, and, at the same time, $\beta_{k}$ is real and independent of $c$.

REMARK 3.1. For definiteness, in the subsequent analysis we will choose the sign + before the radicand in expression $\left(8_{1}\right)$. As is clear, this will not affect a generality of the results.

Taking into account (7), the corresponding surface tractions acting on the plane $\nu \cdot \mathbf{x}=$ $x^{\prime}$ become

$$
\mathbf{t}_{k}\left(x^{\prime}\right)=i r\left(\begin{array}{r}
\left(\begin{array}{r}
\left(\nu \cdot \mathbf{C}_{k} \cdot \nu \otimes \mathbf{m}\right)\left(\alpha_{k} \cosh \left(i r \alpha_{k} x^{\prime}\right)+\beta_{k} \sinh \left(i r \alpha_{k} x^{\prime}\right)\right) \\
+\left(\nu \cdot \mathbf{C}_{k} \cdot \mathbf{n} \otimes \mathbf{m}\right) \sinh \left(i r \alpha_{k} x^{\prime}\right)
\end{array}\right) C_{2 k-1} \\
+\left(\begin{array}{r}
\left(\nu \cdot \mathbf{C}_{k} \cdot \nu \otimes \mathbf{m}\right)\left(\alpha_{k} \sinh \left(i r \alpha_{k} x^{\prime}\right)+\beta_{k} \cosh \left(i r \alpha_{k} x^{\prime}\right)\right) \\
+\left(\nu \cdot \mathbf{C}_{k} \cdot \mathbf{n} \otimes \mathbf{m}\right) \cosh \left(i r \alpha_{k} x^{\prime}\right)
\end{array}\right) C_{2 k}
\end{array}\right) e^{i r\left(\beta_{k} x^{\prime}+\mathbf{n} \cdot \mathbf{x}-c t\right)}
$$

Proposition 3.1. Surface tractions (9) are collinear with vector $\mathbf{m}$.

Proof. The proof flows out from the assumed monoclinic symmetry with respect to sagittal plane, that ensures an even number of entering vector $\mathbf{m}$ in the decomposable components of the tensor $\mathbf{C}_{k}$ (in the orthogonal basis generated by vectors $\mathbf{m}, \nu, \mathbf{n}$ ). Thus, both vectors $\left(\nu \cdot \mathbf{C}_{k} \cdot \nu \otimes \mathbf{m}\right)$ and $\left(\nu \cdot \mathbf{C}_{k} \cdot \mathbf{n} \otimes \mathbf{m}\right)$ in the right-hand side of (9) are collinear with vector $\mathbf{m}$. 
Taking into account (9) and the facts that $\nu \cdot \mathbf{C}_{k} \cdot \mathbf{n} \otimes \mathbf{m}=0, \beta_{k}=0$, and $\gamma_{k}=\alpha_{k}$ for the orthotropic material with axes of elastic symmetry coinciding with vectors $\mathbf{m}, \mathbf{n}$, and $\nu$, we arrive at the following expression for the surface tractions acting in the orthotropic layer:

$$
\mathbf{t}_{k}\left(x^{\prime}\right)=i r \gamma_{k}\left(\nu \cdot \mathbf{C}_{k} \cdots \nu \otimes \mathbf{m}\right)\left(C_{2 k-1} \cosh \left(i r \gamma_{k} x^{\prime}\right)+C_{2 k} \sinh \left(i r \gamma_{k} x^{\prime}\right)\right) e^{i r(\mathbf{n} \cdot \mathbf{x}-c t)} .
$$

3.2. Multiple Roots. Representation (7) for the surface waves in a layer becomes incorrect when multiple roots of the Christoffel equation arise; see [1]. These roots arise when the discriminant of Eq. (6), determined by the parameter $\alpha_{k}$, vanishes. This allows us to formulate the following proposition:

Proposition 3.2. a) The phase speed at which multiple roots arise is

$$
c=\sqrt{\rho^{-1}\left(\mathbf{m} \otimes \mathbf{n} \cdot \mathbf{C}_{k} \cdot \mathbf{n} \otimes \mathbf{m}-\frac{\left(\mathbf{m} \cdot \operatorname{sym}\left(\mathbf{n} \cdot \mathbf{C}_{k} \cdot \nu\right) \cdot \mathbf{m}\right)^{2}}{\mathbf{m} \otimes \nu \cdot \mathbf{C}_{k} \cdot \nu \otimes \mathbf{m}}\right)} .
$$

b) The corresponding Christoffel parameter $\gamma_{k}$ (necessarily real) is

$$
\gamma_{k}=-\frac{\mathbf{m} \cdot \operatorname{sym}\left(\mathbf{n} \cdot \mathbf{C}_{k} \cdot \nu\right) \cdot \mathbf{m}}{\mathbf{m} \otimes \nu \cdot \mathbf{C}_{k} \cdot \nu \otimes \mathbf{m}}
$$

c) Representation for the displacement field corresponding to multiple roots is

$$
\mathbf{u}_{k}(\mathbf{x})=\mathbf{m}\left(C_{2 k-1}+i r x^{\prime} C_{2 k}\right) e^{i r\left(\gamma_{k} x^{\prime}+\mathbf{n} \cdot \mathbf{x}-c t\right)} .
$$

d) The corresponding surface tractions on the plane $\nu \cdot \mathbf{x}=x^{\prime}$ are

$$
\mathbf{t}_{k}\left(x^{\prime}\right)=i r\left(\begin{array}{r}
\left(\nu \cdot \mathbf{C}_{k} \cdot \nu \otimes \mathbf{m}\right)\left(\gamma_{k} C_{2 k-1}+\left(1+i r \gamma_{k} x^{\prime}\right) C_{2 k}\right) \\
+\left(\nu \cdot \mathbf{C}_{k} \cdot \mathbf{n} \otimes \mathbf{m}\right)\left(C_{2 k-1}+i r x^{\prime} C_{2 k}\right)
\end{array}\right) e^{i r\left(\gamma_{k} x^{\prime}+\mathbf{n} \cdot \mathbf{x}-c t\right)}
$$

Proof. Conditions a) and b) flow out from considering the vanishing discriminant in (6). Condition c) corresponds to the general solution of Eq. (5) at multiple roots; see [1].

Proposition 3.3. Surface tractions (14) are collinear with vector $\mathbf{m}$.

Proof. The proof is analogous to the proof of Proposition 3.1.

REMARK 3.2. a) Analysis of expression (11) shows that multiple roots exist, if and only if the material properties of a medium satisfy inequality

$$
\mathbf{m} \otimes \mathbf{n} \cdot \mathbf{C}_{k} \cdot \mathbf{n} \otimes \mathbf{m}>\frac{\left(\mathbf{m} \cdot \operatorname{sym}\left(\mathbf{n} \cdot \mathbf{C}_{k} \cdot \nu\right) \cdot \mathbf{m}\right)^{2}}{\mathbf{m} \otimes \nu \cdots \mathbf{C}_{k} \cdot \nu \otimes \mathbf{m}}
$$

This inequality ensures the phase speed determined by Eq.(11) to be real. Now, it remains to observe that (15) is a direct consequence of positive definite condition (3). Thus, for any positive definite monoclinic medium there is one and only one value of the phase speed, at which multiple roots arise.

b) Expressions (11), (12) reveal that for an orthotropic material having chrystallographical axes collinear with vectors $\mathbf{m}, \mathbf{n}$, and $\nu$, multiple roots arise at

$$
c=c_{\mathbf{m n}}^{T},
$$


where $c_{\mathbf{m n}}^{T}$ is the speed of the shear bulk wave propagating in $\mathbf{n}$ and polarized in the $\mathbf{m}$ direction. Moreover, at this speed the corresponding multiple roots $\gamma_{k}$ vanish.

\section{Modified transfer matrix method.}

4.1. Transfer matrices. According to Propositions 3.1 and 3.2 the scalar amplitudes of the displacements and surface tractions acting in the $k$-th layer on the plane $\nu \cdot \mathbf{x}=x^{\prime}$ can be represented in the form

$$
\left(\begin{array}{l}
u_{k}\left(x^{\prime}\right) \\
t_{k}\left(x^{\prime}\right)
\end{array}\right)=\mathbf{M}_{k}\left(x^{\prime}\right) \cdot\left(\begin{array}{l}
C_{2 k-1} \\
C_{2 k}
\end{array}\right)
$$

where $u_{k}\left(x^{\prime}\right) \equiv\left|\mathbf{u}_{k}\left(x^{\prime}\right) e^{-i r(\mathbf{n} \cdot \mathbf{x}-c t)}\right|, t_{k}\left(x^{\prime}\right) \equiv\left|\mathbf{t}_{k}\left(x^{\prime}\right) e^{-i r(\mathbf{n} \cdot \mathbf{x}-c t)}\right|$ are the corresponding scalar amplitudes, and $\mathbf{M}_{k}$ is a $2 \times 2$ matrix. Taking into account expressions (7), (9), (13), and (14), matrix $\mathbf{M}_{k}$ takes the form:

a) Aliquant roots

$\mathbf{M}_{k}\left(x^{\prime}\right)=\left(\begin{array}{c}(i r)^{-1} \sinh \left(i r \alpha_{k} x^{\prime}\right) \\ \left(a_{k}\left(\begin{array}{c}\alpha_{k} \cosh \left(i r \alpha_{k} x^{\prime}\right) \\ +\beta_{k} \sinh \left(i r \alpha_{k} x^{\prime}\right)\end{array}\right)+b_{k} \sinh \left(i r \alpha_{k} x^{\prime}\right)\right)\end{array}\right) \quad\left(\begin{array}{c}\left.a_{k}\left(\begin{array}{c}\alpha_{k} \sinh \left(i r \alpha_{k} x^{\prime} x^{\prime}\right) \\ +\beta_{k} \cosh \left(i r \alpha_{k} x^{\prime}\right)\end{array}\right)+b_{k} \sinh \left(i r \alpha_{k} x^{\prime}\right)\right)\end{array}\right) i r e^{i r \beta_{k} x^{\prime},}$

where $a_{k}=\mathbf{m} \otimes \nu \cdot \mathbf{C}_{k} \cdot \nu \otimes \mathbf{m}, \quad b_{k}=\mathbf{m} \otimes \nu \cdots \mathbf{C}_{k} \cdot \mathbf{n} \otimes \mathbf{m}$.

b) Multiple roots

$$
\mathbf{M}_{k}\left(x^{\prime}\right)=\left(\begin{array}{cc}
(i r)^{-1} & x^{\prime} \\
\left(\gamma_{k} a_{k}+b_{k}\right) & \left(\left(1+i r \gamma_{k} x^{\prime}\right) a_{k}+i r x^{\prime} b_{k}\right)
\end{array}\right) i r e^{i r \gamma_{k} x^{\prime}} .
$$

Note that according to (8) parameter $\beta_{k}$ in (18) is independent of the phase speed $c$. The following proposition takes place:

Proposition 4.1. For both aliquant and multiple roots matrices $\mathbf{M}_{k}$ are nonsingular at any real $x^{\prime}$.

Proof. The proof can be found in [1].

We should also note that for an orthotropic layer the matrix $\mathbf{M}_{k}$ takes the form:

$$
\mathbf{M}_{k}\left(x^{\prime}\right)=i r\left(\begin{array}{cc}
(i r)^{-1} \sinh \left(i r \gamma_{k} x^{\prime}\right) & (i r)^{-1} \cosh \left(i r \gamma_{k} x^{\prime}\right) \\
a_{k} \gamma_{k} \cosh \left(i r \gamma_{k} x^{\prime}\right) & a_{k} \gamma_{k} \sinh \left(i r \gamma_{k} x^{\prime}\right)
\end{array}\right)
$$

Now, by use of transfer matrices $\mathbf{M}_{k}$, the unknown coefficients $\vec{C}^{(n)} \equiv\left(C_{2 n-1} ; C_{2 n}\right)$ corresponding to $n$-th layer can be expressed in terms of coefficients $\vec{C}^{(1)} \equiv\left(C_{1} ; C_{2}\right)$ related to the first layer:

$$
\vec{C}^{(n)}=\mathbf{M}_{n}^{-1}\left(h_{n} / 2\right) \cdot\left(\prod_{k=2}^{n-1}\left(\mathbf{M}_{k}\left(-h_{k} / 2\right) \cdot \mathbf{M}_{k}^{-1}\left(h_{k} / 2\right)\right)\right) \cdot \mathbf{M}_{1}\left(-h_{1} / 2\right) \cdot \vec{C}^{(1)},
$$

where $h_{k}, k=1, \ldots, n$ is the thickness of the corresponding layer. 
4.2. Traction-free outer surfaces. Expressions (9) and (14) allow us to formulate traction-free boundary conditions in the form

$$
\left\{\begin{array}{l}
t_{1}\left(h_{1} / 2\right) \equiv \vec{T}^{(1)}\left(h_{1} / 2\right) \cdot \vec{C}^{(1)}=0, \\
t_{n}\left(-h_{n} / 2\right) \equiv \vec{T}^{(n)}\left(-h_{n} / 2\right) \cdot \vec{C}^{(n)}=0
\end{array}\right.
$$

where $t_{k}$ are the corresponding scalar amplitudes of the surface tractions; $\vec{T}^{(k)}=$ $\left(X_{k} ; Y_{k}\right), k=1, n$; and components $X_{k}$ and $Y_{k}$ take the form:

a) Aliquant roots

$$
\begin{aligned}
& X_{k}\left( \pm h_{k} / 2\right) \\
& \quad=\left(a_{k}\left(\alpha_{k} \cosh \left(i r \alpha_{k} h_{k} / 2\right) \pm \beta_{k} \sinh \left(i r \alpha_{k} h_{k} / 2\right)\right) \pm b_{k} \sinh \left(i r \alpha_{k} h_{k} / 2\right)\right) i r e^{ \pm i r \beta_{k} h_{k} / 2}, \\
& Y_{k}\left( \pm h_{k} / 2\right) \\
& \quad=\left(a_{k}\left( \pm \alpha_{k} \sinh \left(i r \alpha_{k} h_{k} / 2\right)+\beta_{k} \cosh \left(i r \alpha_{k} h_{k} / 2\right)\right)+b_{k} \cosh \left(i r \alpha_{k} h_{k} / 2\right)\right) i r e^{ \pm i r \beta_{k} h_{k} / 2} .
\end{aligned}
$$

b) Multiple roots

$$
\begin{aligned}
& X_{k}\left( \pm h_{1} / 2\right)=\left(\gamma_{k} a_{k}+b_{k}\right) i r e^{ \pm i r \gamma_{k} h_{k} / 2} \\
& Y_{k}\left( \pm h_{1} / 2\right)=\left(\left(1 \pm i r \gamma_{k} h_{k} / 2\right) a_{k} \pm i r b_{k} h_{k} / 2\right) i r e^{ \pm i r \gamma_{k} h_{k} / 2}
\end{aligned}
$$

Now, boundary condition $(21)_{1}$ allows us to express (up to a multiplier) coefficients $C_{1}$ and $C_{2}$ in terms of components of the vector $\vec{T}^{(1)}$. Introducing orthogonal to $\vec{T}^{(1)}\left(h_{1} / 2\right)$ vector

$$
\vec{T}_{\perp}^{(1)}\left(h_{1} / 2\right)=\left(-Y_{1}\left(h_{1} / 2\right) ; X_{1}\left(h_{1} / 2\right)\right)
$$

and taking

$$
\vec{C}^{(1)}={\overrightarrow{T_{\perp}}}^{(1)}\left(h_{1} / 2\right),
$$

we satisfy boundary condition $(21)_{1}$.

Satisfying boundary condition $(21)_{2}$ can be achieved by taking scalar multiplication of the right-hand side of Eq. (20) with vector $\vec{T}^{(n)}\left(-h_{n} / 2\right)$ and equating it to zero according to $(21)_{2}$. This yields the desired secular equation of the MTM method:

$$
\vec{T}^{(n)}\left(-h_{n} / 2\right) \cdot\left(\mathbf{M}_{n}^{-1}\left(h_{n} / 2\right) \cdot\left(\prod_{k=2}^{n-1}\left(\mathbf{M}_{k}\left(-h_{k} / 2\right) \cdot \mathbf{M}_{k}^{-1}\left(h_{k} / 2\right)\right)\right) \cdot \mathbf{M}_{1}\left(-h_{1} / 2\right)\right) \cdot{\overrightarrow{T_{\perp}}}^{(1)}\left(h_{1} / 2\right)=0 .
$$

4.3. Clamped outer surfaces. These boundary conditions can be expressed in the form:

$$
\left\{\begin{array}{l}
u_{1}\left(h_{1} / 2\right) \equiv \vec{U}^{(1)}\left(h_{1} / 2\right) \cdot \vec{C}^{(1)}=0, \\
u_{n}\left(-h_{n} / 2\right) \equiv \vec{U}^{(n)}\left(-h_{n} / 2\right) \cdot \vec{C}^{(n)}=0,
\end{array}\right.
$$

where $u_{k}$ are scalar amplitudes of the corresponding displacements. According to (7), (13), components of the two-dimensional vectors $\vec{U}^{(k)}=\left(R_{k} ; S_{k}\right) k=1, n$ are:

a) Aliquant roots

$$
\begin{aligned}
& R_{k}\left( \pm h_{k} / 2\right)= \pm \sinh \left(i r \alpha_{k} h_{k} / 2\right) e^{ \pm i r \beta_{k} h_{k} / 2}, \\
& S_{k}\left( \pm h_{k} / 2\right)=\cosh \left(i r \alpha_{k} h_{k} / 2\right) e^{ \pm i r \beta_{k} h_{k} / 2} .
\end{aligned}
$$

b) Multiple roots

$$
\begin{aligned}
& R_{k}\left( \pm h_{k} / 2\right)=e^{ \pm i r \gamma_{k} h_{k} / 2} \\
& S_{k}\left( \pm h_{k} / 2\right)= \pm\left(i r h_{k} / 2\right) e^{ \pm i r \gamma_{k} h_{k} / 2}
\end{aligned}
$$


Introducing orthogonal to $\vec{U}^{(1)}\left(h_{1} / 2\right)$ vector

$$
{\overrightarrow{U_{\perp}}}^{(1)}\left(h_{1} / 2\right)=\left(-S_{1}\left(h_{1} / 2\right) ; R_{1}\left(h_{1} / 2\right)\right),
$$

boundary condition $(27)_{1}$ can be satisfied by taking

$$
\vec{C}^{(1)}={\overrightarrow{U_{\perp}}}^{(1)}\left(h_{1} / 2\right) .
$$

Now, boundary condition $(27)_{2}$ yields the following secular equation that is similar to (26):

$$
\vec{U}^{(n)}\left(-h_{n} / 2\right) \cdot\left(\mathbf{M}_{n}^{-1}\left(h_{n} / 2\right) \cdot\left(\prod_{k=2}^{n-1}\left(\mathbf{M}_{k}\left(-h_{k} / 2\right) \cdot \mathbf{M}_{k}^{-1}\left(h_{k} / 2\right)\right)\right) \cdot \mathbf{M}_{1}\left(-h_{1} / 2\right)\right) \cdot \vec{U}_{\perp}^{(1)}\left(h_{1} / 2\right)=0 .
$$

4.4. Mixed boundary conditions. Herein we consider the traction-free upper surface and clamped bottom surface of the plate:

$$
\left\{\begin{array}{l}
t_{1}\left(h_{1} / 2\right) \equiv \vec{T}^{(1)}\left(h_{1} / 2\right) \cdot \vec{C}^{(1)}=0, \\
u_{n}\left(-h_{n} / 2\right) \equiv \vec{U}^{(n)}\left(-h_{n} / 2\right) \cdot \vec{C}^{(n)}=0 .
\end{array}\right.
$$

For the regarded case the secular equation takes the form

$$
\vec{U}^{(n)}\left(-h_{n} / 2\right) \cdot\left(\mathbf{M}_{n}^{-1}\left(h_{n} / 2\right) \cdot\left(\prod_{k=2}^{n-1}\left(\mathbf{M}_{k}\left(-h_{k} / 2\right) \cdot \mathbf{M}_{k}^{-1}\left(h_{k} / 2\right)\right)\right) \cdot \mathbf{M}_{1}\left(-h_{1} / 2\right)\right) \cdot{\overrightarrow{T_{\perp}}}^{(1)}\left(h_{1} / 2\right)=0 .
$$

Modification of Eq. (34) for the case, when upper surface is clamped and the bottom is traction-free, is obvious.

REMARK 4.1. The left-hand sides of secular equations (26), (32), and (34) can be regarded as the implicit equations with respect to the wave number $r$ (at the fixed phase frequency $\omega$ ). Using in these equations the relation

$$
r=\frac{\omega}{c},
$$

we arrive at the implicit equations yielding the dispersion relations in terms of the frequency $\omega$ and phase speed.

\section{Homogeneous (single-layered) plate.}

5.1. Plate with traction-free boundary conditions. For such a plate the secular equations (26), (32), and (34) can be considerably simplified by excluding the transfer matrices. This yields

$$
\vec{T}^{(1)}\left(-h_{1} / 2\right) \cdot{\overrightarrow{T_{\perp}}}^{(1)}\left(h_{1} / 2\right)=0,
$$

where $h_{1}$ is the thickness of the plate. Substituting components $X_{1}, Y_{1}$ into (36) yields

$$
X_{1}\left(h_{1} / 2\right) Y_{1}\left(-h_{1} / 2\right)-Y_{1}\left(h_{1} / 2\right) X_{1}\left(-h_{1} / 2\right)=0 .
$$

a) Aliquant roots.

Proposition 5.1. At the aliquant roots: (i) there are dispersion surface waves satisfying the dispersion relation

$$
\omega=\frac{n \pi c}{\alpha_{1} h_{1}}, \quad n=1,2, \ldots
$$


where the parameter $\alpha_{1}$ is defined by $(8)_{1}$; (ii) the dispersion relation (38) is valid, if and only if the phase speed belongs to the interval

$$
\begin{aligned}
& c \in\left(c_{0} ; \infty\right), \\
& c_{0} \equiv \sqrt{\frac{1}{\rho_{1}}\left(\left(\mathbf{m} \otimes \mathbf{n} \cdot \mathbf{C}_{1} \cdot \mathbf{n} \otimes \mathbf{m}\right)-\frac{\left(\mathbf{m} \cdot \mathbf{s y m}\left(\mathbf{n} \cdot \mathbf{C}_{1} \cdot \nu\right) \cdot \mathbf{m}\right)^{2}}{\left(\mathbf{m} \otimes \nu \cdot \cdot \mathbf{C}_{1} \cdot \nu \nu \mathbf{m}\right)}\right.} .
\end{aligned}
$$

Proof. Proof of (i) flows out from Eq. (37) with the help of (22), (35), and Remark 3.1. The proof of (ii) follows from Eq. (8) $)_{1}$, since condition (39) ensures the parameter $\alpha_{1}$ to be real, otherwise the phase frequency becomes complex.

REMARK 5.1. a) The right-hand side of (39) shows that the lower bound $c_{0}$ for possible velocities of the regarded waves depends upon material properties. This bound may coincide with the shear bulk wave speed $c_{m n}^{T}$ for an arbitrary orthotropic plate with $\mathbf{m} \cdot \operatorname{sym}\left(\mathbf{n} \cdot \mathbf{C}_{1} \cdot \nu\right) \cdot \mathbf{m}=0$, or it may be subsonic for a monoclinic plate with $\mathbf{m} \cdot \operatorname{sym}\left(\mathbf{n} \cdot \mathbf{C}_{1} \cdot \nu\right) \cdot \mathbf{m} \neq 0$.

b) Analysis of Eq. (37) reveals that along with the dispersion surface waves satisfying Eq. (38), there can be another apparent non-dispersion and non-homogeneous horizontally polarized shear wave propagating with the phase speed

$$
c=\sqrt{\frac{\mathbf{m} \otimes \mathbf{n} \cdot \mathbf{C}_{1} \cdot \mathbf{n} \otimes \mathbf{m}-\frac{\left(\mathbf{m} \otimes \nu \cdot \cdot \mathbf{C}_{1} \cdot \mathbf{n} \otimes \mathbf{m}\right)\left(\mathbf{m} \otimes \mathbf{n} \cdot \mathbf{C}_{1} \cdot \nu \otimes \mathbf{m}\right)}{\mathbf{m} \otimes \nu \cdot \cdot \mathbf{C}_{1} \cdot \nu \otimes \mathbf{m}}}{\rho_{1}}},
$$

at which Eq. (37) is satisfied. But Eq. (40) yields exactly the same value for the phase speed as Eq. (11) for appearing multiple roots. So, the regarded apparent solution should be excluded at this stage, as related to multiple roots.

b) Multiple roots.

At multiple roots Eq. (37) along with Eqs. (12), (23), and (35) yield:

Proposition 5.2. At multiple roots there is one horizontally polarized non-dispersion shear wave satisfying traction-free boundary conditions; such a wave exists for any monoclinic plate with positive definite elasticity tensor (see Remark 3.2a).

REMARK 5.2. If the elasticity tensor belongs to the rhombic (orthotropic) system and the crystallographical axes are collinear with vectors $\mathbf{m}, \mathbf{n}$ and $\nu$, then the regarded non-dispersion wave turns out to be the shear bulk wave.

5.2. Clamped plate. For a plate with clamped boundary conditions the secular equation takes the form

$$
\vec{U}^{(1)}\left(-h_{1} / 2\right) \cdot{\overrightarrow{U_{\perp}}}^{(1)}\left(h_{1} / 2\right)=0,
$$

where vectors $\vec{U}^{(1)},{\overrightarrow{U_{\perp}}}^{(1)}$ were introduced in Section 4.3. Substituting components $R_{1}, S_{1}$ into (41) yields

$$
R_{1}\left(h_{1} / 2\right) S_{1}\left(-h_{1} / 2\right)-S_{1}\left(h_{1} / 2\right) R_{1}\left(-h_{1} / 2\right)=0 .
$$

a) Aliquant roots.

With help of (28) and (35), Eq. (42) yields:

Proposition 5.3. At the aliquant roots: (i) there are dispersion surface waves satisfying dispersion relation (38); (ii) dispersion relation (38) is valid, if and only if the phase speed satisfies condition (39). 
b) Multiple roots.

For multiple roots Eq. (42) along with (12), (29), and (35) lead to the following proposition:

Proposition 5.4. At multiple roots no horizontally polarized surface waves propagate.

5.3. Plate with mixed boundary conditions. For such a plate the secular equation takes the form

$$
\vec{U}^{(1)}\left(-h_{1} / 2\right) \cdot{\overrightarrow{T_{\perp}}}^{(1)}\left(h_{1} / 2\right)=0,
$$

where it is assumed that the upper surface is traction-free, while the bottom surface is clamped. Substituting components $R_{k}, S_{k}$ and $X_{k}, Y_{k}$ into (43) yields

$$
S_{1}\left(-h_{1} / 2\right) X_{1}\left(h_{1} / 2\right)-R_{1}\left(-h_{1} / 2\right) Y_{1}\left(h_{1} / 2\right)=0 .
$$

a) Aliquant roots.

Analysis of Eq. (44), taking into account (8), (22), and (28), allows us to formulate:

Proposition 5.5. At the aliquant roots no horizontally polarized waves propagate.

b) Multiple roots.

Similar to Proposition 5.4, we have:

Proposition 5.6. At multiple roots no horizontally polarized waves propagate.

REMARK 5.3. a) Summarizing, we arrive at the following characterization of propagating ability for the regarded SH-waves: (i) for a traction-free plate there are both dispersion (with the unbounded speed interval defined by (39)) and non-dispersion waves; (ii) for a clamped plate only dispersion waves can propagate (for this case the speed interval coincides with (39)); and (iii) for a plate with mixed boundary conditions no wave can propagate. Thus, to cut off the non-dispersion wave it is needed to clamp both surfaces of a traction-free plate, while clamping one surface eliminates both of these waves.

b) The presented analysis also reveals that for all the considered boundary conditions the phase speed and frequency are delimited from zero. This fact results in the absence of either horizontally polarized stationary waves or waves with infinite wavelength.

c) Expression (38) allows us to obtain the lowest limiting frequencies $\omega_{\text {lim }}$ attained at $c \rightarrow \infty$ for both traction-free and clamped plates:

$$
\omega_{\lim }=\frac{n \pi c_{\mathbf{m} \nu}^{T}}{h_{1}}, \quad n=1,2, \ldots,
$$

where $c_{\mathbf{m} \nu}^{T}$ is the speed of the transverse bulk wave propagating in $\nu$ and polarizing in the $\mathbf{m}$ direction.

d) Analysis of expressions (8) and (38) reveals that at $c \rightarrow c_{0}+0$ ( $c_{0}$ was defined by (39)), the corresponding frequencies become unbounded.

6. Two-layered plate with orthotropic layers. Herein we assume that the crystallographical axes of both layers are collinear, and vectors $\mathbf{m}, \mathbf{n}$ and $\nu$ coincide with these axes. 
6.1. Traction-free plate. According to Eq. (26) the secular equation for such a plate becomes

$$
\vec{T}^{(2)}\left(-h_{2} / 2\right) \cdot \mathbf{M}_{2}^{-1}\left(h_{2} / 2\right) \cdot \mathbf{M}_{1}\left(-h_{1} / 2\right) \cdot \vec{T}_{\perp}^{(1)}\left(h_{1} / 2\right)=0 .
$$

Taking into account Eqs. (18) and (22), composition in the left-hand side of Eq. (46) yields up to a multiplier $i r^{2} a_{1} \gamma_{1}$ :

$$
a_{1} \gamma_{1} \sin \left(\omega \gamma_{1} h_{1} / c\right) \cos \left(\omega \gamma_{2} h_{2} / c\right)+a_{2} \gamma_{2} \cos \left(\omega \gamma_{1} h_{1} / c\right) \sin \left(\omega \gamma_{2} h_{2} / c\right)=0,
$$

where indices 1 and 2 refer to the corresponding layers. Equation (47) is the resulting equation we are looking for.

Proposition 6.1. a) Equation (47) admits no solutions at the phase speed

$$
c \in\left(0 ; \min \left(\left(c_{\mathbf{m n}}^{T}\right)_{1} ;\left(c_{\mathbf{m n}}^{T}\right)_{2}\right)\right) .
$$

b) There are dispersion solutions at the phase speed belonging to the interval

$$
c \in\left(\min \left(\left(c_{\mathbf{m n}}^{T}\right)_{1} ;\left(c_{\mathbf{m n}}^{T}\right)_{2}\right) ; \infty\right) .
$$

c) There is a non-trivial dispersion solution at the phase frequency $\omega \rightarrow 0$ (the phase frequency is not delimited from zero).

Proof. a) At the phase speed belonging to the interval (48), Eq. (47) transforms into

$$
\frac{\tanh \left(i \omega \gamma_{1} h_{1} / c\right)}{\tanh \left(i \omega \gamma_{2} h_{2} / c\right)}=-\frac{a_{2} \gamma_{2}}{a_{1} \gamma_{1}},
$$

but the latter equation has no solutions at positive $\omega$ and $c$, because the left- and righthand sides of Eq. (50) have different signs (arguments at tanh-functions are real, since both $\gamma_{1}$ and $\gamma_{2}$ are imaginary).

To prove b) it is sufficient to present solutions corresponding to simultaneous vanishing of either sine or cosine functions in (47). This gives the following values for the phase speed:

$$
\begin{aligned}
& c=\sqrt{\frac{a_{1} d_{2} h_{2}^{2} m^{2}-a_{2} d_{1} h_{1}^{2} n^{2}}{a_{1} \rho_{2} h_{2}^{2} m^{2}-a_{2} \rho_{1} h_{1}^{2} n^{2}}}, \quad c=\sqrt{\frac{a_{1} d_{2} h_{2}^{2}-a_{2} d_{1} h_{1}^{2}+4 a_{1} d_{2} h_{2}^{2} m(1+m)-4 a_{2} d_{1} h_{1}^{2} n(1+n)}{a_{1} \rho_{2} h_{2}^{2}-a_{2} \rho_{1} h_{1}^{2}+4 a_{1} \rho_{2} h_{2}^{2} m(1+m)-4 a_{2} \rho_{1} h_{1}^{2} n(1+n)}}, \\
& n, m \in \mathbb{Z}_{+}
\end{aligned}
$$

where the first solution corresponds to vanishing sine functions and the other one to cosine. Integers $n$ and $m$ are chosen in such a way that the phase speed belongs to the interval (49). In (51) we denote $d_{k}=\mathbf{m} \otimes \mathbf{n} \cdot \mathbf{C}_{k} \cdot \mathbf{n} \otimes \mathbf{m}$. At these values of the phase speed, the corresponding frequencies become

$$
\omega=\frac{n \pi c}{\gamma_{1} h_{1}}, \quad \omega=\frac{(1 / 2+n) \pi c}{\gamma_{1} h_{1}}, \quad n \in \mathbb{Z}_{+} .
$$

The Christoffel parameter $\gamma_{1}$ in (52) is real due to considering speed interval. It should also be noted that there could be other kinds of solutions, not related to the vanishing of either sine or cosine functions.

c) First, we observe that function $F(\omega, c)$ in the left-hand side of Eq. (47) is analytic with respect to both $c \in\left(\min \left(\left(c_{\mathbf{m n}}^{T}\right)_{1} ;\left(c_{\mathbf{m n}}^{T}\right)_{2}\right) ; \infty\right)$ and $\omega \in(-\infty, \infty)$, and it is odd with respect to $\omega$. The latter condition does not allow us to construct the implicit function $c(\omega)$ directly at $\omega=0$. But we are able to define an even and differentiable at 
$\omega=0$ branch of this implicit function (an odd and continuous at $\omega=0$ branch cannot exist due to Proposition 6.1a). Applying the implicit function theorem to the even branch $c(\omega)$ yields

$$
\partial_{\omega} F(\omega, c)=0, \text { at } \omega=0 .
$$

Equation (53) delivers a necessary condition for $c^{\prime}(0)=0$. Solving Eq. (53) yields the phase speed $c_{s}$ at which Eq. (53) is satisfied:

$$
c_{s}=\sqrt{\frac{\left(\mathbf{m} \otimes \mathbf{n} \cdot \mathbf{C}_{1} \cdot \mathbf{n} \otimes \mathbf{m}\right) h_{1}+\left(\mathbf{m} \otimes \mathbf{n} \cdot \mathbf{C}_{2} \cdot \mathbf{n} \otimes \mathbf{m}\right) h_{2}}{\rho_{1} h_{1}+\rho_{2} h_{2}}} .
$$

Thus, in contrast to the preceding section, for a two-layered traction-free plate the phase frequency is not delimited from zero.

REMARK 6.1. a) Direct verification shows that

$$
\min \left(\left(c_{\mathbf{m n}}^{T}\right)_{1} ;\left(c_{\mathbf{m n}}^{T}\right)_{2}\right)<c_{s}<\left(c_{\mathbf{m n}}^{T}\right)_{1}+\left(c_{\mathbf{m n}}^{T}\right)_{2}
$$

provided $\left(c_{\mathbf{m n}}^{T}\right)_{1} \neq\left(c_{\mathbf{m n}}^{T}\right)_{2}$.

b) Probably the most interesting observation is concerned with the last condition of Proposition 6.1. In fact, it ensures existence of the $\mathrm{SH}$-wave propagating with the finite speed $c_{s}$ at vanishing phase frequency; see also [5]-7]. Thus, the regarded branch of the SH-wave at the speed $c_{s}$ must have an infinite wavelength resembling a solitary wave. It should also be noted that for Love waves there exists a similar solution at the phase speed approaching the shear bulk wave speed in the substrate, but such a wave as being leakage (it does not attenuate with depth in the substrate) is of limited interest.

c) Another observation concerns the same speed interval and the existence of the considered SH-waves at any admissible physical properties of the layers. In this respect these waves differ from the genuine Love wave, which exists only if $\left(c_{\mathbf{m n}}^{T}\right)_{\text {layer }}<\left(c_{\mathbf{m n}}^{T}\right)_{\text {substrate }}$; see [1].

6.2. Clamped plate. For such a plate the secular equation takes the form

$$
\vec{U}^{(2)}\left(-h_{2} / 2\right) \cdot \mathbf{M}_{2}^{-1}\left(h_{2} / 2\right) \cdot \mathbf{M}_{1}\left(-h_{1} / 2\right) \cdot{\overrightarrow{U_{\perp}}}^{(1)}\left(h_{1} / 2\right)=0 .
$$

Taking into account Eqs. (18) and (28), composition in the left-hand side of Eq. (55) yields up to a multiplier $\left(i r^{2} a_{1} \gamma_{1}\right)$ :

$$
a_{2} \gamma_{2} \sin \left(\omega \gamma_{1} h_{1} / c\right) \cos \left(\omega \gamma_{2} h_{2} / c\right)+a_{1} \gamma_{1} \cos \left(\omega \gamma_{1} h_{1} / c\right) \sin \left(\omega \gamma_{2} h_{2} / c\right)=0 .
$$

Proposition 6.2. a) Equation (56) admits no solutions at the phase speed belonging to the interval (48).

b) There are dispersion solutions at the phase speed belonging to the interval

$$
c \in\left(\min \left(\left(c_{\mathbf{m n}}^{T}\right)_{1} ;\left(c_{\mathbf{m n}}^{T}\right)_{2}\right) ; \infty\right) .
$$

c) There is no non-trivial dispersion solution at the phase frequency $\omega \rightarrow 0$ (the phase frequency is delimited from zero).

Mainly, the proof is analogous to the proof of Proposition 6.1, except Proposition 6.2c. To prove the latter it is sufficient to demonstrate that no even branch of the implicit function $c(\omega)$ exists in the vicinity of $\omega=0$. Due to the implicit function theorem this is equivalent to condition (53). From (53) we obtain the following two values of the phase speed: $\left(c_{\mathbf{m n}}^{T}\right)_{1}$ and $\left(c_{\mathbf{m n}}^{T}\right)_{2}$, at which this equation is satisfied, but the subsequent analysis 
reveals that no continuous branches $c(\omega)$ at small $\omega$ correspond to these values of the phase speed.

6.3. Plate with mixed boundary conditions. For such a plate the secular equation takes the form

$$
\vec{U}^{(2)}\left(-h_{2} / 2\right) \cdot \mathbf{M}_{2}^{-1}\left(h_{2} / 2\right) \cdot \mathbf{M}_{1}\left(-h_{1} / 2\right) \cdot \vec{T}_{\perp}^{(1)}\left(h_{1} / 2\right)=0 .
$$

Equation (58) corresponds to the clamped bottom surface. Taking into account Eqs. (18) and (34), composition in the left-hand side of Eq. (58) yields up to a multiplier $i r^{2} a_{1} \gamma_{1}$ :

$$
-a_{2} \gamma_{2} \cos \left(\omega \gamma_{1} h_{1} / c\right) \cos \left(\omega \gamma_{2} h_{2} / c\right)+a_{1} \gamma_{1} \sin \left(\omega \gamma_{1} h_{1} / c\right) \sin \left(\omega \gamma_{2} h_{2} / c\right)=0 .
$$

Proposition 6.3. a) Equation (59) admits no solutions at the phase speed belonging to the interval (48).

b) There are dispersion solutions at the phase speed belonging to the interval (57).

c) There is no non-trivial dispersion solution at the phase frequency $\omega \rightarrow 0$ (the phase frequency is delimited from zero).

Proof. The proof is analogous to the proof of Proposition 6.2.

REMARK 6.2. Again, as was pointed out in Remark 6.1c, the considered SH-waves exist at any admissible values of physical properties of the layers.

7. Three-layered traction-free plate with orthotropic layers. Herein we consider a three-layered plate whose outer layers have equal physical properties and thickness. All these layers are assumed to be orthotropic with the crystallographical axes coinciding with vectors $\mathbf{m}, \mathbf{n}$ and $\nu$. According to Eq. (26) the secular equation for such a plate becomes

$$
\vec{T}^{(1)}\left(-h_{1} / 2\right) \cdot \mathbf{M}_{1}^{-1}\left(h_{1} / 2\right) \cdot \mathbf{M}_{2}\left(-h_{2} / 2\right) \cdot \mathbf{M}_{2}^{-1}\left(h_{2} / 2\right) \cdot \mathbf{M}_{1}\left(-h_{1} / 2\right) \cdot \vec{T}_{\perp}{ }^{(1)}\left(h_{1} / 2\right)=0 .
$$

We recall that both outer layers have the same properties denoted by index 1 ; index 2 refers to the internal layer. Taking into account Eqs. (18) and (22), composition in the left-hand side of Eq. (60) yields up to a multiplier $i r \frac{a_{1} \gamma_{1}}{a_{2} \gamma_{2}}$ :

$$
\begin{aligned}
& a_{1} \gamma_{1} a_{2} \gamma_{2} \sin \left(2 \omega \gamma_{1} h_{1} / c\right) \cos \left(\omega \gamma_{2} h_{2} / c\right) \\
& \quad+\left(a_{2}^{2} \gamma_{2}^{2} \cos ^{2}\left(\omega \gamma_{1} h_{1} / c\right)-a_{1}^{2} \gamma_{1}^{2} \sin ^{2}\left(\omega \gamma_{1} h_{1} / c\right)\right) \sin \left(\omega \gamma_{2} h_{2} / c\right)=0 .
\end{aligned}
$$

Equation (61) is the resulting equation we are looking for.

Proposition 7.1. a) Equation (61) admits no solutions at the phase speed

$$
c \in\left(0 ; \min \left(\left(c_{\mathbf{m n}}^{T}\right)_{1} ;\left(c_{\mathbf{m n}}^{T}\right)_{2}\right)\right) .
$$

b) There are dispersion solutions at the phase speed belonging to the interval

$$
c \in\left(\min \left(\left(c_{\mathbf{m n}}^{T}\right)_{1} ;\left(c_{\mathbf{m n}}^{T}\right)_{2}\right) ; \infty\right) .
$$

c) There is a non-trivial dispersion solution at the phase frequency $\omega \rightarrow 0$ propagating with the speed

$$
c_{s}=\sqrt{\frac{2\left(\mathbf{m} \otimes \mathbf{n} \cdot \mathbf{C}_{1} \cdots \mathbf{n} \otimes \mathbf{m}\right) h_{1}+\left(\mathbf{m} \otimes \mathbf{n} \cdot \mathbf{C}_{2} \cdots \mathbf{n} \otimes \mathbf{m}\right) h_{2}}{2 \rho_{1} h_{1}+\rho_{2} h_{2}}}
$$


(the phase frequency is not delimited from zero).

Proof. The proof is analogous to the proof of Proposition 6.1.

REMARK 7.1. a) Direct verification shows that

$$
\min \left(\left(c_{\mathbf{m n}}^{T}\right)_{1} ;\left(c_{\mathbf{m n}}^{T}\right)_{2}\right)<c_{s}<\left(c_{\mathbf{m n}}^{T}\right)_{1}+\left(c_{\mathbf{m n}}^{T}\right)_{2},
$$

provided $\left(c_{\mathbf{m n}}^{T}\right)_{1} \neq\left(c_{\mathbf{m n}}^{T}\right)_{2}$.

b) Proposition 7.1 ensures existence of the $\mathrm{SH}$-waves in the regarded traction-free plate at any admissible properties of both outer and internal layers.

c) Assuming that the dimensionless parameter $\frac{\omega \gamma_{2} h_{2}}{c}$ is real, and $\frac{\omega \gamma_{2} h_{2}}{c} \rightarrow 0$, or $h_{2} \rightarrow 0$ (very thin internal layer) at $\frac{\omega \gamma_{2}}{c}=O\left(h_{2}\right)$, then Eq. (61) yields

$$
\omega=\frac{n \pi c}{2 \gamma_{1} h_{1}}, \quad n=1,2, \ldots,
$$

where the phase speed $c$ is necessarily greater than $\max \left(\left(c_{\mathbf{m n}}^{T}\right)_{1} ;\left(c_{\mathbf{m n}}^{T}\right)_{2}\right)$. It is interesting to note that at $\frac{\omega \gamma_{2} h_{2}}{c} \rightarrow 0$ physical properties of the internal layer are not essential, though this layer should remain solid with the non-vanishing shear module and density.

d) Assuming $\frac{\omega \gamma_{1} h_{1}}{c}$ is real, and $\frac{\omega \gamma_{1} h_{1}}{c} \rightarrow 0$, or $h_{1} \rightarrow 0$ (very thin outer layers) at $\frac{\omega \gamma_{1}}{c}=O\left(h_{1}\right)$, then Eq. (61) yields

$$
\omega=\frac{n \pi c}{\gamma_{2} h_{2}}, \quad n=1,2, \ldots,
$$

where the phase speed should exceed $\max \left(\left(c_{\mathbf{m n}}^{T}\right)_{1} ;\left(c_{\mathbf{m n}}^{T}\right)_{2}\right)$. As in the preceding remark, the corresponding physical properties of the outer layers are not essential.

Acknowledgements. The author thanks the Russian Foundation for Basic Research (Grant 04-01-00781) and the RF President Program for Scientific Schools (Grant 1849.2003.1) for financial support.

\section{REFERENCES}

[1] S. V. Kuznetsov, Love waves in stratified monoclinic media, Quart. Appl. Math. 62, 749-766 (2004). MR2104272 (2005g:74067)

[2] A. E. H. Love, Some Problems of Geodynamics, Cambridge University Press, London, 1911.

[3] H. Lamb, On waves in an elastic plate, Proc. Roy. Soc. A93, 114-128 (1917).

[4] A. L. Shuvalov, On the theory of wave propagation in anisotropic plates, Proc. Roy. Soc. A456, 2197-2222 (2000). MR1794722 (2001h:74042)

[5] A. H. Nayfeh, The propagation of horizontally polarized waves in multilayered anisotropic media, J. Acoust. Soc. Am., 86(5) 2000-2012 (1989).

[6] A. H. Nayfeh, Wave propagation in layered anisotropic media with application to composites, Elsevier, Amsterdam, 1995. MR 1356003 (96j:73028)

[7] F. Simonetti and P. Cawley, A guided wave technique for the characterization of highly attenuative viscoelastic materials, J. Acoust. Soc. Am., 114(1), 158-165 (2003). 